\title{
Modelling of Projectile Penetration into Lightweight Expanded Clay Aggregate
}

\author{
Juha Kuutti ${ }^{1}$
}

Received: 2 June 2016/Accepted: 7 September 2016/Published online: 12 September 2016

(C) Society for Experimental Mechanics, Inc 2016

\begin{abstract}
This work presents a phenomenological model for estimating projectile penetration into granular lightweight expanded clay aggregate often known as Lightweight expanded clay aggregate (LECA). Laboratory scale penetration experiments in the low-velocity regime performed using LECA are presented. A penetration model based on the mass and momentum balances is proposed and the model parameters are derived from the physical behaviour of the penetrated material. It was found that the penetration behaviour predicted by the model agrees reasonably well with the experimental results for three cases studied in detail in terms of projectile deceleration during the penetration and the final penetration depth as well as for the other cases for which only measured penetration depths were available. Although the proposed model results correspond well with the experiments, further validation and more detailed modelling of the dynamic effects of the assumed deformation mechanisms of the penetrated material are needed.
\end{abstract}

Keywords Lightweight expanded clay aggregate (LECA) · Penetration experiments · Crushing · Phenomenological model

Juha Kuutti

juha.kuutti@vtt.fi

1 VTT Technical Research Centre of Finland, Kemistintie 3, P.O. Box 1000, 02044 Espoo, Finland

\section{Introduction}

This work presents a phenomenological model for estimating projectile penetration into granular lightweight expanded clay aggregate often known as Lightweight expanded clay aggregate (LECA). The presented penetration experiments and the assumptions made in formulating the penetration model focus on this granular material type.

First, the performed laboratory scale experimental impact testing of a rigid projectile into the LECA material in an impact velocity regime below $100 \mathrm{~m} / \mathrm{s}$ accompanied by the material characterisation testing are summarised. These dynamic penetration tests were originally performed for the design and dimensioning of a shock absorber filled with a granular material. The experiments were first presented by the current author in a research report [1]. The selected tests from the full test campaign are considered in this work in more detail. The material considered for the shock absorber purposes at that time was LECA with the grain size in the range of $0-10 \mathrm{~mm}$. The performed penetration tests are similar to the tests reported in Ref. [2] but with a higher impact velocity and a horizontal setting. The common uses for LECA are in manufacturing lightweight concrete and geotechnical applications. The author is not aware of any other impact resistance or dynamic penetration tests performed using clay aggregates as the target material. However, lightweight concrete manufactured using similar clay aggregates has been used in impact resistance studies for instance in Refs. [3-5]. Several projectile penetration studies have been performed for other types of granular materials, especially sand [6-13].

In this work, the common phenomenological models that can be used to predict projectile penetration behaviour are discussed and their ability to predict the penetration 
resistance and resulting penetration depth in the current case is evaluated. A new Newton's second law based penetration model that utilises the concepts of the Riera model developed originally for aircraft impacts [14] is then presented. The proposed model includes the contributions from the particle crushing and compaction and friction on the penetration resistance. The model can be simplified to be consistent with the common models for projectile penetration developed by Robins-Euler, Poncelet and Résal [15-17]. Using the analogy with the Riera model, the model parameters are derived from the physical properties of the penetrated granular LECA material.

The results obtained with the developed model are compared in detail against the experimentally measured deceleration during the penetration in three cases where such measurements were available. In addition, the penetration depths predicted by the model are compared against the corresponding experimental data for the other cases of the experimental programme.

\section{Compaction, Shear and Penetration Resistance of LECA}

LECA is a commercial lightweight granular material [18] manufactured by expanding natural clay in a rotary kiln by heating. The rotating manufacturing process produces pitted round granules that have a hard ceramic shell and a highly porous structure. The typical use of the material is in manufacturing lightweight concrete as well as in geotechnical fillings. The typical grain sizes used in these applications are in the region of $0-32 \mathrm{~mm}$ and bulk density of $200-500 \mathrm{~kg} / \mathrm{m}^{3}$. According to the manufacturer [18], the density of the clay used to manufacture the LECA granules is $2610 \mathrm{~kg} / \mathrm{m}^{3}$ and the bulk porosity (voids between the granules) of the LECA is around $40 \%$, depending on the size distribution. When submerged, the water content in the granules increases to $30 \%$ and can increase significantly over time.

The penetration resistance of LECA was experimentally studied as it was planned to be the shock absorbing material for a safety shock absorber device in a spent nuclear fuel repository. The spent nuclear fuel from the nuclear power plants in Finland will be disposed of in an underground repository excavated in bedrock in Olkiluoto in Western Finland. The 25 ton metal disposal canisters containing the spent fuel are planned to be transferred to the repository located at the depth of $400-450 \mathrm{~m}$ by a vertical lift. During the design of the repository, an excavated pit filled with granular material was considered as a shock absorbing device below the lift shaft for disposal canister falling accidents. The granular LECA material was initially considered for the shock absorber use due to its wide availability, inorganic and non-flammable nature and relatively low penetration resistance that would cause tolerable deceleration magnitudes for the disposal canister. The laboratory scale penetration tests summarised in this work were performed for the dimensioning purposes of such shock absorber device [1].

In the original research reported in Ref. [1], the behaviour of the granular LECA material was characterised by uniaxial static compression and direct shear tests. The shock absorbing capability was studied with dynamic penetration tests. In the experimental programme, the penetration resistance of different grain sizes of the LECA material was studied in three different scales. In this work, the tests using the LECA type with trade name KS04 and grain size of $0-4 \mathrm{~mm}$ are considered in detail because the wireless deceleration measurements succeeded for this LECA type. The tests performed with the LECA type with trade name KS410 and grain size of $4-10 \mathrm{~mm}$ are also considered.

\section{Compaction Resistance}

The static uniaxial compression experiments were performed to determine the confined compressive resistance of the granular LECA material. In the tests, a material sample with the height of $100 \mathrm{~mm}$ was uniaxially and quasi-statically compressed in a rigid container with a rigid indenter nearly equal to the container diameter of $64 \mathrm{~mm}$. The performed test procedure utilised the concepts of the testing standard EN 13055-1 [19] that can be used to characterise lightweight aggregates. The piston is forced into the cylinder causing compression in the material sample. The magnitude of the compressive force or pressure together with the compressive displacement or the change of material sample volume is used to quantify the crushing resistance. The standard defines the compaction resistance as a single compressive pressure value for a specified magnitude of compressive displacement. In the current approach, the complete measured compaction force and compressive displacement history transformed into compressive pressures and volumetric strains were used to model the compaction behaviour. A schematic drawing of the test setup, a virgin material sample, a compressed sample and obtained pressure-compaction curve are presented in Fig. 1. The presented data was measured for material samples with grain size $0-4 \mathrm{~mm}$. During the compression, the individual LECA granules first fracture and the resulting fine sand compresses even further. Due to the fracture and relocation of the granules and sand, the unloading stiffness and permanent deformation are high as can be seen from the test data labelled KSO4 sample 3. The specific details of the uniaxial testing are presented in Ref. [1]. 


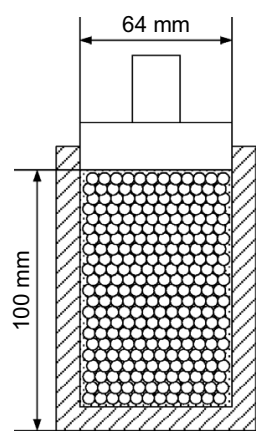

(a)

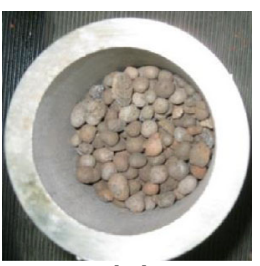

(b)

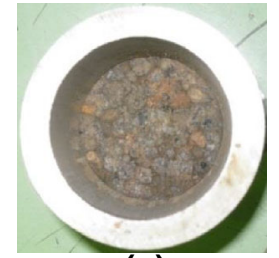

(c)

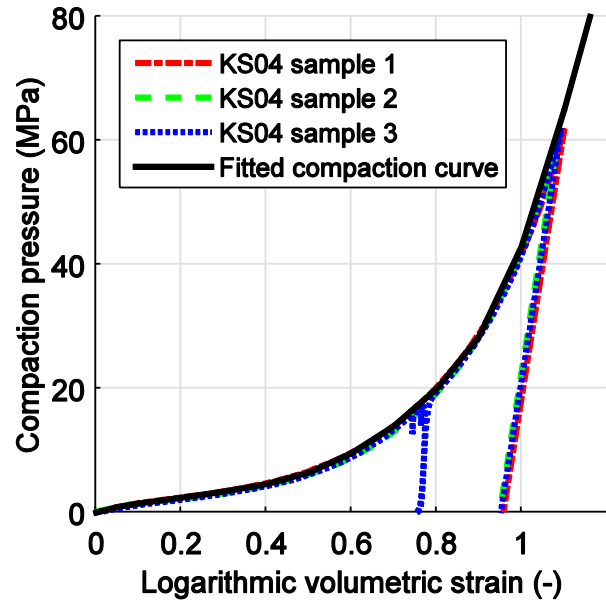

(d)

Fig. 1 Uniaxial compressive testing set-up of the granular LECA material with grain size $0-4 \mathrm{~mm}(\mathbf{a})$, material samples before (b) and after compression (c) and obtained compaction curves (d)

\section{Shear Resistance}

The direct shear tests were performed to evaluate the shear properties of the granular LECA material. The utilised test set-up was a direct shear test procedure that produces a shear plane in the middle of the material sample thus measuring the shear resistance and internal friction of the material. In accordance with the standard direct shear test procedure [20], the shear strength of the material samples was measured using several confining pressures. The friction angle was determined by plotting the maximum shear stress of each test as a function of the confining pressure and determining the slope of the best fit straight line through the data points. The intersection of the best fit line at zero confinement indicates the amount of cohesion in the material. These values calculated from the test results are presented in Table 1. Also, the effect of watering the material sample was studied in the experiments but it appeared to have no significant influence on the shear resistance. The bulk density of the material was also measured for both dry and watered conditions. The averages of the bulk densities measured in the before-testing state are presented in Table 1. The "dry" conditions indicate that the sample was as stored in the laboratory facilities with a normal room temperature and humidity. The "watered" conditions indicate that the sample was put into water just before the testing and the excess water was then drained with a fine sieve such that only the water absorbed by the granules remain in the specimen. The "soaked" conditions indicate that the material was kept approximately 1 month in water before draining the excess water. The measured densities show that the water content increases quickly but does not increase significantly during the first 30 days of soaking.

\section{Penetration Resistance}

The penetration tests for the granular LECA material were carried out in three different scales at the VTT Technical Research Centre of Finland laboratory facilities during 2011 and 2012 [1]. In these tests, a rigid small scale projectile representative of the spent nuclear fuel disposal canister was shot into a LECA filled target pipe in a horizontal setting. The main test results in the current work were obtained with a blunt-nosed steel projectile with a mass of $5.3 \mathrm{~kg}$ and diameter of $63 \mathrm{~mm}$ shot into the target pipe with a diameter of $400 \mathrm{~mm}$ and length of $4000 \mathrm{~mm}$ filled with LECA KS04 material with grain size of 0-4 $\mathrm{mm}$. The initial impact velocities in all of the tests were in the range from 40 to $90 \mathrm{~m} / \mathrm{s}$. The LECA type KS410 with grain size of $4-10 \mathrm{~mm}$ was also used in the experimental programme. A schematic drawing of the test setup is shown in Fig. 2 and photographs of the target pipe and projectile are shown in Fig. 3. In the tests, the projectile was shot using compressed air horizontally into the target, which was a non-deforming cylindrical pipe with rolling supports and a smooth inner surface containing the

Table 1 Summary of the results from the direct shear tests with LECA KS04 with grain size of 0-4 mm and the corresponding bulk densities

\begin{tabular}{llll}
\hline Sample conditions & Bulk density $\left(\mathrm{kg} / \mathrm{m}^{3}\right)$ & Cohesion $(\mathrm{kPa})$ & Mohr-Coulomb friction angle \\
\hline KS04, dry (no additional watering) & 570 & 4.6 & $37.9^{\circ}$ \\
KS04, watered before test & 693 & $\sim 0$ & $38.8^{\circ}$ \\
KS04, soaked in water & 741 & 4.6 & $37.6^{\circ}$ \\
\hline
\end{tabular}



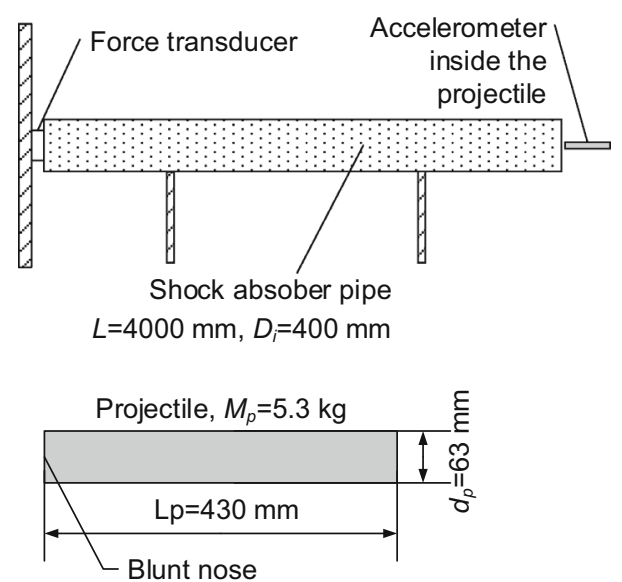

Fig. 2 Schematic drawing of the LECA penetration test setup
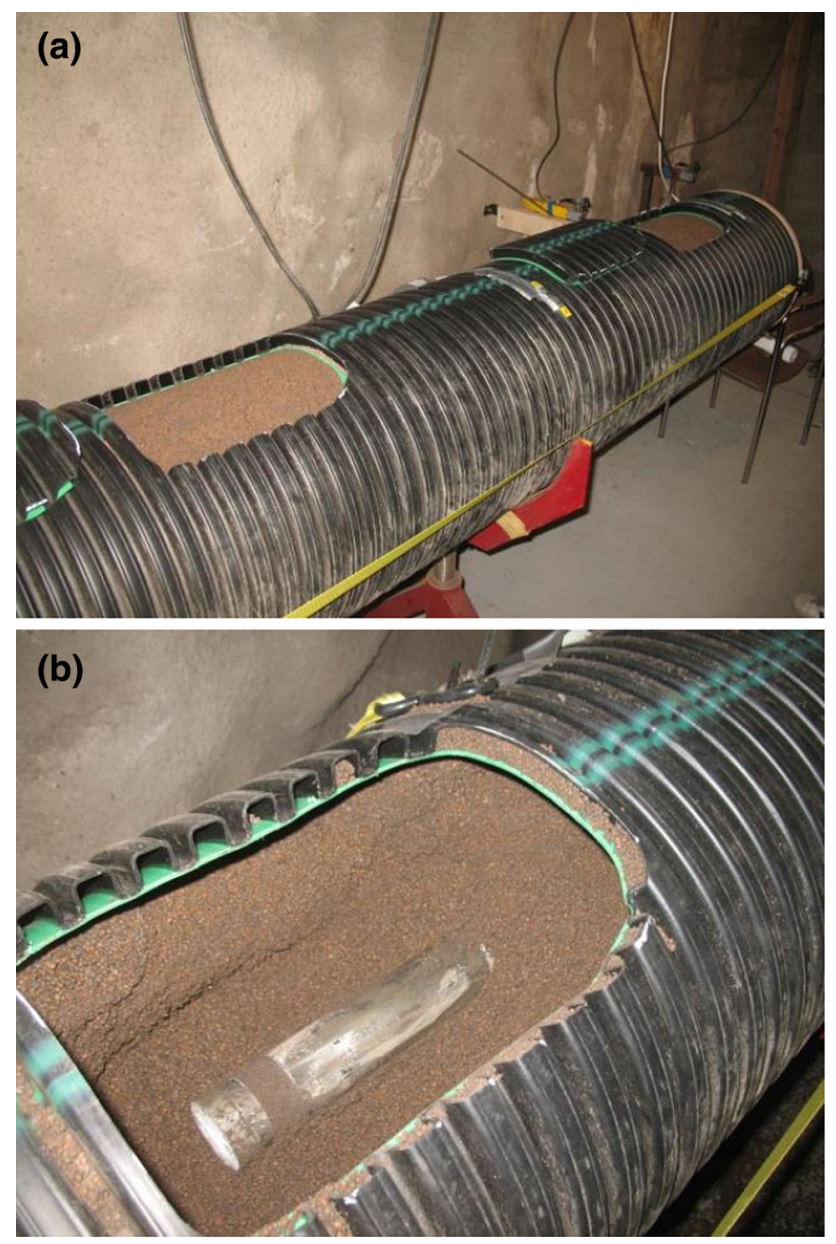

Fig. 3 The target pipe (a) and projectile among the LECA granules after a penetration test (b)

shock absorbing material. The pipe was filled with the LECA material using a low-pressure air pump and the target material was watered with a hose in some tests. The ends of the pipe were loose enough to let the excess water drain out.

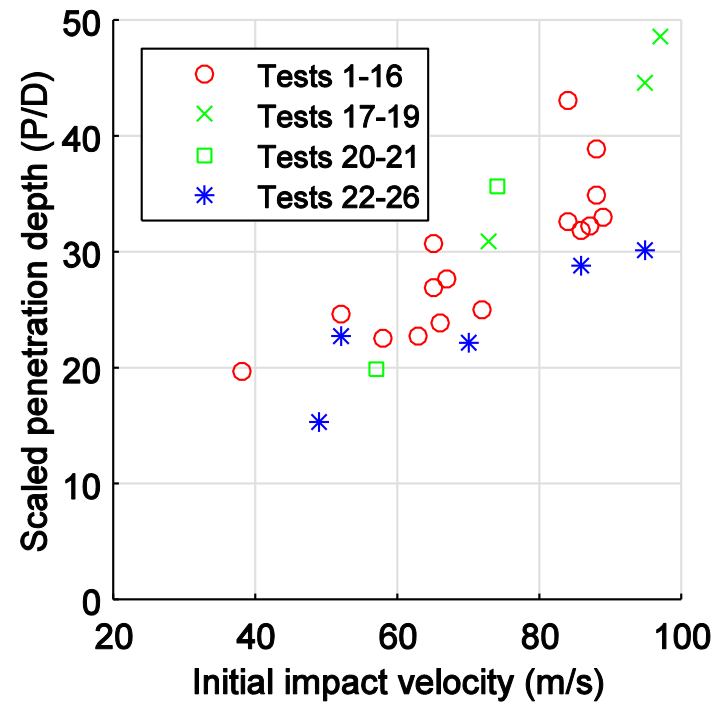

Fig. 4 Scaled penetration depths measured in all of the LECA penetration tests. The measured penetration is scaled with the projectile diameter

The deceleration of the projectile during the penetration was measured using an in-house developed wireless accelerometer installed inside the projectile. Laser detectors were used to measure the velocity of the projectile just prior to the impact. The internal pressure in the target and movement of the projectile was approximated by the strain gauges glued circumferentially on the pipe external surface. The overall impact momentum used to assess the possible rebound of the projectile was obtained from the force transducer installed behind the pipe at the wall support. The final penetration depth after each test was measured by hand with a straight rod from the front of the target and the measurement was confirmed when the projectile was dug out.

The penetration depths measured in each test are presented as a function of initial impact velocity in Fig. 4. The numbering of the tests relates to the test conditions. The tests 1-21 were performed using LECA KS410 with grain size of $4-10 \mathrm{~mm}$ as the target material and the tests $22-26$ using LECA KS04 with the smaller grain size of 0-4 mm. The tests numbered as 17-21 were performed using larger projectiles and target pipes. The diameters of the projectiles were 112 and $150 \mathrm{~mm}$ and the target pipe was scaled accordingly. The tests $22-26$ were performed with the smaller projectile according to the description above and the tests $23-25$ are considered in this work in detail. In the first 14 tests, the penetrated material was not watered and the target pipe was only filled and mixed between the tests. Therefore, there were some remains of broken LECA granules within the virgin material in these tests. Sand resulting from the broken LECA granules was found after each test from the trajectory of the projectile. The order of 
the tests compared with the numeric data of the penetration depths indicates that there was no systematic increase or decrease in penetration caused by not completely changing the target material. A compacted portion of the crushed material was found in front of the projectile in some of the tests. Although not measured in detail, the largest dimension of the irregularly shaped plug was typically in the vicinity of the projectile diameter.

In the tests studied in this work in detail, the target material was fully changed for each test and watered to simulate the ground water of the bedrock disposal facility. The results indicate that the effect of the watering of the target material cannot be quantified from the other scatter of the test results. The experiments with the smaller grain size target material produced smaller penetration values than the respective tests with the larger grain size, but the number of the tests is not sufficient for actual statistical considerations. The manual filling of the target pipe and the resulting possible differences in the packing and composition of the target material can be identified as the main source of the scatter in the penetration depths. The difference of the target material water content may also affect the scatter in the tests 1-16.

The wireless accelerometer inside the projectile functioned correctly in the tests $23-25$. The impact velocity measured with the laser sensors and penetration depth measured after the test could be calculated with reasonable accuracy from the acceleration-deceleration signal. An example of the typical measured deceleration signal and the integrated projectile velocity and penetration curves are shown in Fig. 5. The presented data was measured in the test 23 with the impact velocity of $70 \mathrm{~m} / \mathrm{s}$.

\section{Methods for Predicting Projectile Penetration}

Phenomenological models based on Newton's second law have been applied to model projectile penetration into granular media [15]. The resistance to penetration $F$ as a function of the projectile velocity $v$ offered by the penetrated material is often resolved into the three components:

$F=\left(\alpha v^{2}+\beta v+\gamma\right) A_{p}$,

where $\alpha$ represents the contribution of the inertial forces, $\beta$ represents the magnitude of the first-order viscous resistance (i.e. drag) and $\gamma$ is a constant resistance related to the static penetration strength of the material. As these parameters can be considered as characteristics of the penetrated material, they are defined per unit area and the head area of the projectile $A_{p}$ is included in the equation. As summarised in Ref. [15], the penetration magnitude can be derived from this resistance equation using different assumptions for the parameters of the penetrated material.
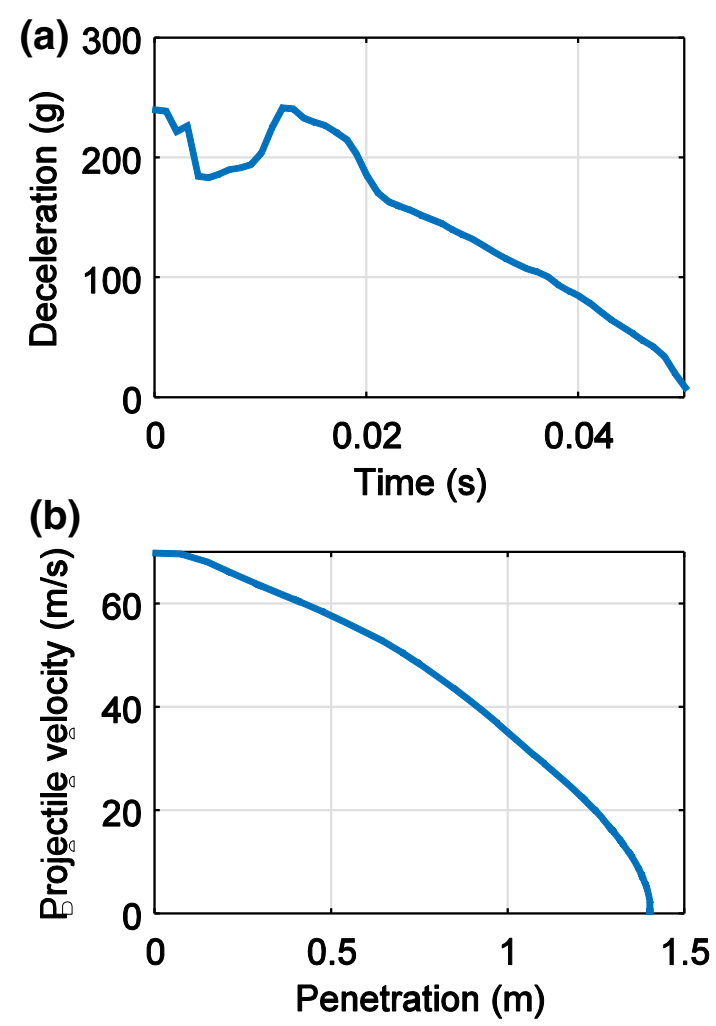

Fig. 5 Measured projectile deceleration as a function of time (a) and the integrated projectile velocity as a function of projectile penetration (b). The data was measured in the test 23

For example, the widely utilised Poncelet penetration equation assumes that $\beta=0$ and the penetration resistance is caused only by the inertia and static resistance of the penetrated material.

Penetration models of this form have been employed for non-cohesive granular materials by several authors [9-11, 21-25]. Although the penetrated material consists of macroscopic particles, the phenomenological models consider the material as a continuum with separable inertial, viscous and strength properties. The properties then correspond to the effects of both particle-particle interactions and projectile-particle interaction. The calibration of the parameters for the penetration resistance equation is often performed only using the experimental penetration studies. Experiments performed in different scales or velocity regimes are studied to gain more confidence in the assumed deformation mechanisms. As a result, the parameters calibrated in this fashion are not necessarily the explicit properties of the penetrated material but dependent also on the projectile and experimental conditions. The penetration models based on the cavity expansion analysis are also applied in describing the response of the penetrated material and predicting the projectile penetration behaviour $[15,26,27]$. The cavity produced by the projectile during penetration gives rise to the stresses and strains that are in 
turn modelled by a constitutive relationship of the penetrated material. For a selected constitutive model, the penetration resistance predicted by the cavity expansion analysis can be written in the form of Eq. (1). The parameters are then more related to the physical properties and deformation mechanisms of the penetrated material. In addition to these phenomenological or analytical techniques, numerical continuum [7, 28, 29] or discrete based methods $[13,30,31]$ have been applied in modelling projectile penetration. Although much more laborious to perform, the numerical methods allow the more detailed considerations of issues such as particle crushing, nonuniform projectile-particle contact and projectile erosion that are difficult to model using analytical approaches.

In this paper, the efforts are focused in developing a penetration resistance model consistent with the full form of Eq. (1) for the LECA penetration experiments. After initial considerations with the different forms of this equation, it was found that the equation is not able to predict the observed penetration behaviour even in the qualitative sense if the parameters are taken as constants throughout the penetration. Instead of developing casespecific penetration depth and projectile velocity dependent relations for the parameters, mechanical considerations of the penetrated material deformation mechanisms and the penetration process are applied to relate the parameters to the actual physical behaviour and characteristics of the penetrated granular LECA material. This is presented in the following chapter.

\section{Penetration Model with Particle Crushing and Compaction}

The penetration model presented in this chapter is derived based on the momentum and mass considerations of the impacting projectile and the deforming penetrated material. It is assumed that the projectile is rigid and maintains its original orientation and trajectory during the impact. The penetration resistance is assumed to be caused by the inertia and compaction of the penetrated material and the friction between the projectile and the penetrated material. In addition, the total mass of the penetrated target is considered far greater than the mass of the projectile.

\section{Derivation}

The general form of the penetration resistance presented in Eq. (1) is derived by considering the momentum and mass balances of the impacting projectile and penetrated material crushing and compacting in front of the projectile as shown in Fig. 6. The presented derivation of the resistance equation is similar to the derivation of the Riera model that

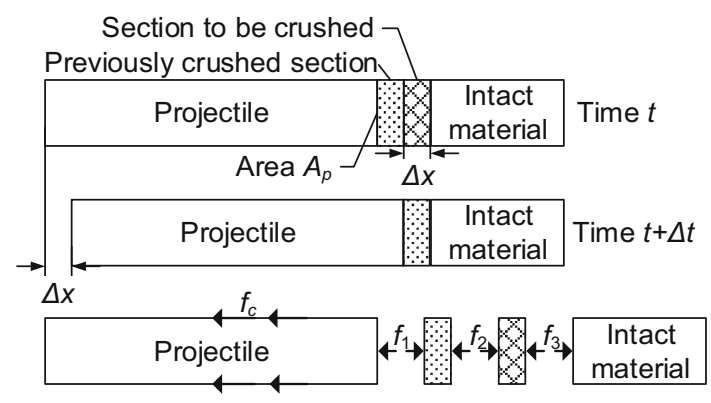

Fig. 6 Schematic drawing of crushing and compaction of the section $\Delta x$ and forces acting on the considered bodies used in the derivation of the penetration resistance model

models the deformation of a soft missile striking a rigid target presented in Ref. [32]. According to the Riera model [14], the force acting on a rigid target caused by a deformable projectile comprises of an inertia related term that accounts for the change of momentum of a small section of the projectile in contact with the target decelerating from the projectile velocity to the target velocity and a crushing related term that is the force required to deform the section of the projectile that crushes and compacts. In the current case of a rigid projectile and deforming target, the inertia term accounts for the change of momentum of a small volume of the penetrated material that accelerates from a stationary state to the velocity of the projectile and the compaction term is the force required to compress this small volume of the penetrated material. In addition, a friction force acting directly on the projectile is included in the model. More formally, the model can be derived based on the momentum and mass balances of the projectile and the crushed and compacted material as follows.

Following the notations of Fig. 6, the penetrated material crushes of the length $\Delta x$ in the time instant $\Delta t$. As the projectile is considered as rigid, the distance travelled by the projectile at the velocity $v_{p}$ equals to the length $\Delta x=v_{p} \Delta t$. During the considered time instant, the increase of the mass of the crushed and compacted material $\Delta M_{c}$ equals to the decrease of the mass of the section to be crushed $\Delta M_{t b c}$ with the density $\rho$ :

$\Delta M_{c}=-\Delta M_{t b c}=\rho A_{p} \Delta x$,

where $A_{p}$ is the head area of the projectile that is assumed to be equal to the cross-sectional area of the section to be crushed.

It is assumed that the projectile and intact material outside the crushing region do not lose mass during the penetration. During the considered increment $\Delta t$, the previously crushed section gains the mass lost by the section to be crushed. Thus, the balance of momentum for the projectile, previously crushed, to be crushed and intact sections read 


$$
\begin{aligned}
& \left(M_{p}+\Delta M_{p}\right)\left(v_{p}+\Delta v_{p}\right)-M_{p} v_{p} \\
& \left(M_{c}+\Delta M_{c}\right)\left(v_{c}+\Delta v_{c}\right)-M_{c} v_{c}=\left(f_{1}-f_{2}\right) \Delta t \\
& \left(M_{t b c}+\Delta M_{t b c}\right)\left(v_{t b c}+\Delta v_{t b c}\right)-M_{t b c} v_{t b c}=\left(f_{2}-f_{3}\right) \Delta t \\
& \left(M_{i}+\Delta M_{i}\right)\left(v_{i}+\Delta v_{i}\right)-M_{i} v_{i}
\end{aligned}
$$

where the forces acting on the projectile and penetrated material are as denoted in Fig. 6. The last equation is excluded from the considerations as it is assumed that the intact material is stationary $\left(v_{i}=0\right)$ and its mass $M_{i}$ is far greater than the mass of the projectile and crushed sections. This equation would in fact model the rigid body motion of an unsupported target material. The other assumptions made in the derivation are that the previously crushed section travels with the projectile, $v_{c}=v_{p}$, the section to be crushed is at rest at the beginning of the increment, $v_{t b c}=0$, and the projectile mass is constant $\Delta M_{p}=0$. The section to be crushed is assumed to be crushed completely, $\Delta M_{t b c}=-M_{t b c}$. With these assumptions and by inserting the mass balance of Eq. (2) we obtain the equations

$$
\begin{aligned}
& M_{p} \Delta v_{p}=-\left(f_{1}+f_{f}\right) \Delta t \\
& \rho A_{p} \Delta x v_{p}+\left(M_{c}+\rho A_{p} \Delta x\right) \Delta v_{p}=\left(f_{1}-f_{2}\right) \Delta t \\
& \left(\rho A_{p} \Delta x-\rho A_{p} \Delta x\right) \Delta v_{t b c}
\end{aligned}
$$

Dividing by $\Delta t$ and by changing the difference notation to derivatives and denoting the static force acting on the intact material as the compaction resistance, $f_{3}=f_{c}$, the system reduces to the form

$$
\rho A_{p} v_{p}^{2}+f_{f}+f_{c}=-\left(M_{p}+M_{c}+\rho A_{p} \Delta x\right) \dot{v}_{p} .
$$

The Eq. (5) controls the motion of the projectile. The force acting on the projectile is then obtained as

$$
\begin{aligned}
f_{p} & =f_{1}+f_{f}=-M_{p} \dot{v}_{p} \\
& =\frac{M_{p}}{M_{p}+M_{c}+\rho A_{p} \Delta x}\left(\rho A_{p} v_{p}^{2}+f_{f}+f_{c}\right) .
\end{aligned}
$$

The bracketed terms in Eq. (6) correspond to the inertial resistance and the crushing and friction forces during the penetration. Their direct effect on the projectile is weakened by the compacted mass accumulating in front of the projectile. If assuming that the crushed mass does not stick to the projectile after crushing $\left(M_{c}=\Delta M_{c}=0\right)$, the model reduces to a form in agreement with both the Poncelet form and the Riera model, $F=\alpha v^{2}+\gamma$. The main difference with the result of the current derivation is that the effective mass of the projectile increases during the penetration as more crushed and compacted mass travel with the projectile.

The above formulation assumes that all crushed and compacted mass remains in motion with the projectile. This may overestimate the size of the plug or false cone formed in front of the projectile. Therefore, the maximum volume of the compacted region in motion with the projectile is limited to a half-sphere with the diameter equal to the projectile diameter. In other words, the compacted region is assumed to form a hemispherical nose to the projectile. The density of the crushed material in the nose is assumed to be the fully compacted density of the target material. Typically, the plug is accumulated during the first phases of penetration and after the plug has been completely formed, the subsequently crushed material is assumed to flow to the wake around the projectile. In the formulation, this affects the mass increase term in Eq. (6) such that the mass increment $\rho A_{p} \Delta x$ is set to zero after the plug has been formed. The inertial resistance due to compaction is present also after the plug formation. The effect of the size of the plug on the penetration resistance is evaluated later in this work.

The initial kinetic energy of the projectile is dissipated through the work done by the penetration resistance. The dissipated energy can be calculated from the work done on the projectile by the frictional and crushing forces. In addition, energy is converted to the kinetic energy of the compacted material in front of the projectile. This energy is lost from the system if the plug has been completely formed and the crushed particles flow to the wake around the projectile.

\section{Compaction and Friction Resistance}

In the traditional Poncelet penetration model, the static resistance is taken as constant $[15,16]$. Typically in the Riera model it is assumed that the static resistance depends on the crushing length [32] $\Delta x$. For the considered granular LECA material, the static resistance comprises of the force required to crush and compact the material in front of the projectile and the frictional resistance caused by the contact between the projectile lateral area and penetrated material.

The crushing and compaction model adapted in this work is motivated based on the experimental observations of a subsonic compaction and damage front located in front of the projectile reported for granular sand in Ref. [33]. In the reference, a constant velocity was given for the compaction wave that arranges the granules into a denser state in front of the projectile. The damage wave that fractures the granules was found to remain near the nose of the projectile. Similar observations on the deformation localised in the vicinity of the projectile nose region can be made from the penetrated sand displacement plots obtained with high-speed imaging and image correlation techniques reported in Ref. [8]. The penetration simulations using particle modelling reported in the work as well confirm this behaviour. In the current model, the compaction resistance 


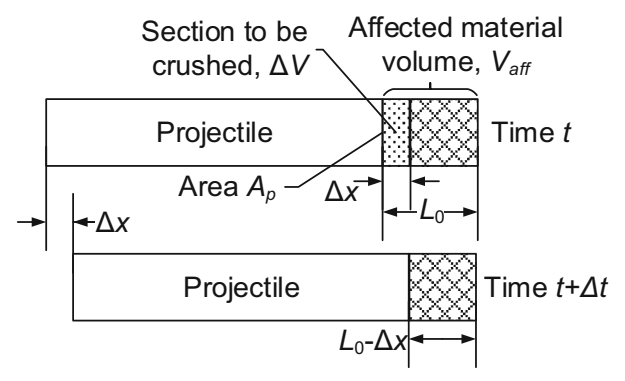

Fig. 7 Determination of the compaction volumetric strain

$f_{c}$ is modelled as a relationship between the compression and volumetric strain. The volumetric strain is calculated by assuming that the material in front of the projectile of the volume $V_{\text {aff }}$ compacts by the amount defined by the projectile movement $\Delta V=A_{p} \Delta x$. The affected material volume $V_{\text {aff }}$ is defined by the speed of subsonic compressive stress wave propagation in the material as $V_{a f f}=A_{p \text { - }}$ $L_{0}=A_{p} v_{0}^{c} \Delta t$. The determination of the compaction volumetric strain is visualised in Fig. 7.

The logarithmic volumetric strain caused by the compaction is obtained as

$$
\begin{aligned}
\varepsilon_{v o l}^{T} & =\ln \left(1-\frac{\Delta V}{V_{a f f}}\right)=\ln \left(1-\frac{A_{p} \Delta x}{A_{p} L_{0}}\right) \\
& =\ln \left(1-\frac{v_{p} \Delta t}{v_{0}^{c} \Delta t}\right)=\ln \left(1-\frac{v_{p}}{v_{0}^{c}}\right)
\end{aligned}
$$

The compaction pressure $p_{c}\left(\varepsilon_{v o l}^{T}\right)$ is evaluated from the measured compression-volumetric strain curve presented in Fig. 1 and the compression resistance is calculated with $f_{c}=p_{c} A_{p}$. The model behaves such that at the initial stages of the penetration the volumetric strain is large which yields to considerable compressive resistance. At the later stages, when the projectile has decelerated, the volumetric strain and compaction resistance are small.

The frictional resistance of the material $f_{f}$ is assumed to be modelled by the Coulomb friction model with the idealised pressure distribution presented in Fig. 8. In the model, the assumed constant lateral pressure $p_{0}$ acting on the rear section of the projectile produces shear stresses. Their magnitude is determined by the lateral pressure and the friction coefficient between the projectile and the penetrated material. This assumption is based on the observations $[8,15]$ that the penetrated material pushed laterally

Fig. 8 Pressure distribution acting on the lateral area of the projectile during penetration aside by the projectile comes into contact only with the rear section of the projectile due to the inertial effects.

The total friction force is obtained by the integration of the shear stress as

$f_{f}=\int_{A_{L}} \tau d A=\mu p_{0} \eta L_{p} \pi d_{p}=\mu p_{0} \eta A_{L}$,

where $\tau$ is the friction shear stress according to the Coulomb friction model with the friction coefficient $\mu . d_{p}, L_{p}$ and $A_{L}$ are the diameter, length and lateral area of the projectile, respectively. The parameters related to the pressure distribution $\eta$ and $p_{0}$ are defined in Fig. 8. The assumed lateral pressure distribution produces a simple form of the total friction resistance, where the resistance is modelled by two parameters that characterise the constant lateral pressure during the penetration and the relative extent of the area the pressure acts on. The area where the friction force acts is determined from the full lateral area of the projectile, or during the initial phases of the penetration, determined by the section that has penetrated into the material. This area can be zero at the very early stages of the penetration.

\section{Resulting Total Penetration Resistance}

Based on the derivations presented above, the total penetration resistance is divided into three separate mechanisms:

$F=\rho A_{p} v_{p}^{2}+f_{c}\left[v_{p}, v_{0}^{c}, p_{c}\left(\varepsilon_{v o l}^{T}\right)\right]+f_{f}\left[\left(p_{0}, \mu, \eta\right)\right]$.

The obtained penetration resistance includes the inertial effects [ $\alpha$-term in Eq. (1)], the term related to the viscous resistance [similar to the $\beta$-term in Eq. (1)] and the resistance independent of the penetration velocity and distance [ $\gamma$-term in Eq. (1)].

The parameters dependent on the penetrated material of this model are the density $\rho$, compressive wave speed $v_{0}^{c}$ and the compaction pressure as a function of volumetric strain $p_{c}\left(\varepsilon_{\text {vol }}^{T}\right)$. The parameters dependent on the interaction are the friction coefficient between the penetrated material and projectile $\mu$, the constant lateral pressure during the penetration $p_{0}$ and the relative extent of the lateral pressure $\eta$.

\section{Application of the Model for LECA Penetration Experiments}

The developed model outlined above is first applied in studying the three tests numbered as $23-25$ in "Compaction, Shear and Penetration Resistance of LECA" section, where the specific details of the test setup were presented. For these tests, the calculated projectile deceleration is compared with the experimental results. In 
addition, the calculated penetration depths are compared with the measured values for the other tests in the full experimental programme.

\section{Model Parameters}

The parameters of the model having a direct physical meaning are the initial bulk density and compaction behaviour of the target material, which are fixed to their experimentally measured values. As the material was watered prior to testing, the density is taken to correspond to the "watered" state in Table 1 . The fully compacted density of the target material is taken as given by the LECA manufacturer [18].

The skin friction coefficient between the steel projectile and LECA material was not directly measured and is approximated based on the measured internal friction angle and literature values for steel-sand skin friction. The internal friction coefficient that sets the upper bound for the skin friction is estimated as 0.8 based on the shear test results presented in "Shear Resistance" section. Friction coefficients between 0.3 and 0.9 are reported in literature for sand-steel and rock-steel interfaces with the higher values obtained for finer grain sizes, higher surface roughness and fast loading rates [34-36]. In the calculations, the value of 0.8 was utilised as the loading rate is fast and the granules fracture into fine sand in front of the projectile.

The parameters calibrated based on the penetration data are the compressive stress wave velocity in the LECA material and lateral pressure related parameters $p_{0}$ and $\eta$. All of the parameters related to the penetrated material and projectile-LECA interaction utilised in the calculations are presented in Table 2 and the constants related to the test setup are presented in Table 3. The numerical integration of Eq. (5) was performed using the explicit central difference integration rule with the time step of $1 \times 10^{-6} \mathrm{~s}$.

\section{Comparison of Projectile Deceleration and Penetration}

The deceleration, velocity and penetration curves presented in Fig. 9 are calculated using the parameters presented
Table 3 Constants related to the test setup for the studied penetration tests

\begin{tabular}{lll}
\hline Parameter & Value & Unit \\
\hline Projectile mass $\left(M_{p}\right)$ & 5.3 & $\mathrm{~kg}$ \\
Projectile diameter $\left(d_{p}\right)$ & 63 & $\mathrm{~mm}$ \\
Projectile length $\left(L_{p}\right)$ & 430 & $\mathrm{~mm}$ \\
Initial impact velocity $\left(v_{0}\right)$ & 70,86 and 95 & $\mathrm{~m} / \mathrm{s}$ \\
\hline
\end{tabular}

above. In the same figures, the experimentally measured data from the three separate tests (denoted as tests 23-25 in "Compaction, Shear and Penetration Resistance of LECA" section) are plotted. The comparison is made in terms of velocity and deceleration as the actual resistance cannot be accurately quantified from the measured deceleration due to the accumulation of the crushed mass in front of the projectile.

Figure $9 \mathrm{~b}$ shows that the model predicts somewhat higher deceleration at the initial stages of the impact than the measured signal. This causes the calculated velocitypenetration curves in Fig. 9a to be slightly below the experimental values. Figure $9 \mathrm{~b}$ also separates the calculated crushing and friction resistance from the total resistance. The point when the projectile has fully penetrated into the material can be seen from the knee point in the friction curve that denotes the contribution of the frictional resistance to total deceleration. It can be seen that at the initial stages, the contribution from the inertial forces accounts for more than half of the resistance but reduces as the penetration progresses and, at the final stages, the resistance is governed only by the compaction and friction components. The frictional resistance increases until the projectile has fully entered the penetrated material and maintains a constant value afterwards. The resistance due to compaction decreases during penetration as the projectile velocity decreases. At the final stages of the penetration, the friction resistance is the only notable component.

An example of the dimensionless energy balance during the penetration is presented for the initial impact velocity of $95 \mathrm{~m} / \mathrm{s}$ in Fig. 10. The energy balance shows that, for the current set of the parameters, approximately $47 \%$ of
Table 2 Parameters calibrated for the LECA material for the studied penetration tests

\begin{tabular}{lll}
\hline Parameter & Value & Unit \\
\hline Target material initial bulk (porous) density $(\rho)$ & 693 & $\mathrm{~kg} / \mathrm{m}^{3}$ \\
Target material compacted (non-porous) density & 2610 & $\mathrm{~kg} / \mathrm{m}^{3}$ \\
Compressive wave speed in the penetrated material $v_{0}^{c}$ & 575 & $\mathrm{~m} / \mathrm{s}$ \\
Compaction of the penetrated material $p_{c}\left(\varepsilon_{v o l}^{T}\right)$ & Curve presented in Fig. 1 \\
Friction coefficient between the projectile and penetrated material $(\mu)$ & 0.8 & - \\
Constant lateral pressure during penetration $\left(p_{0}\right)$ & 0.038 & $\mathrm{MPa}$ \\
Relative extent of the lateral pressure $(\eta)$ & 0.8 & - \\
\hline
\end{tabular}


(a)

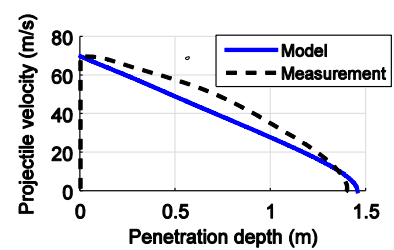

(b)

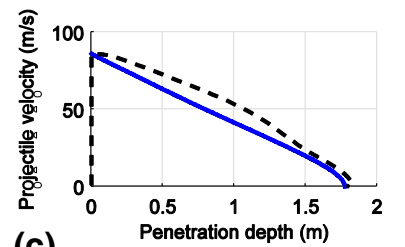

(c)

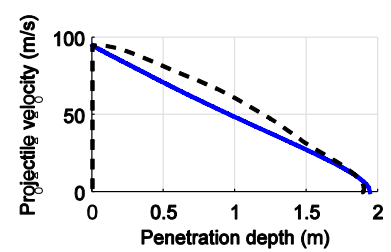

(d)

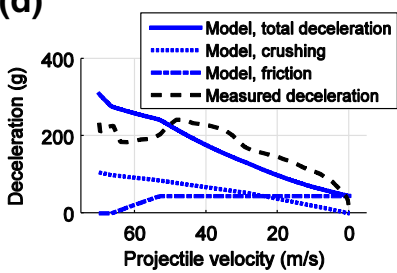

(e)

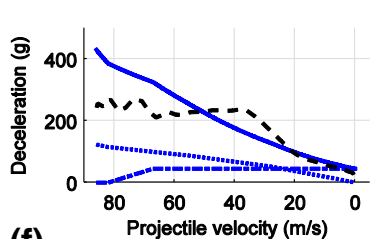

(f)

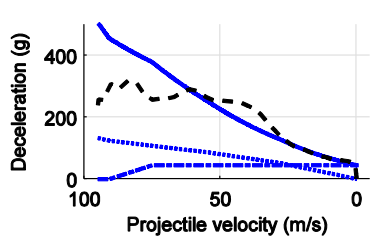

Fig. 9 Projectile velocity as a function of penetration $(\mathbf{a}-\mathbf{c})$ and projectile deceleration as a function of velocity $(\mathbf{d}-\mathbf{f})$ calculated with the developed model for the initial impact velocities of $70 \mathrm{~m} / \mathrm{s}$ (top), $86 \mathrm{~m} / \mathrm{s}$ (mid) and $95 \mathrm{~m} / \mathrm{s}$ (bottom) compared with the deceleration measured in the LECA penetration tests $23-25$ with the same initial impact velocities

the impact energy is dissipated in the kinetic energy of the target material that does not remain with the projectile. The dissipations due to crushing and friction are around 33 and $17 \%$ of the initial impact energy, respectively. The compacted section traveling with the projectile accounts for $3 \%$ of the initial impact energy but this value diminishes as the projectile velocity decreases. As predicted by Eq. (5), the mass accumulating in front of the projectile increases the final penetration depth as the energy transferred from the kinetic energy of the projectile to the kinetic energy of the crushed and compacted section is not lost from the system. However, this effect is relatively weak due to the small volume and mass of the assumed hemispherical nose when compared to the projectile.

\section{Comparison of Penetration Depths}

In addition to the three tests studied above in detail, the developed model is tested by calculating the final penetration depth for each of the tests presented in Fig. 4. The tests 22-26 performed using LECA type KS04 with grain size of $0-4 \mathrm{~mm}$ as the target material are modelled using the parameters presented above. For calculation of the tests 1-21, the target material bulk density and parameters related to the compaction behaviour were changed to correspond to the experimentally determined properties [1] of the LECA type KS410 with grain size of 4-10 mm used in

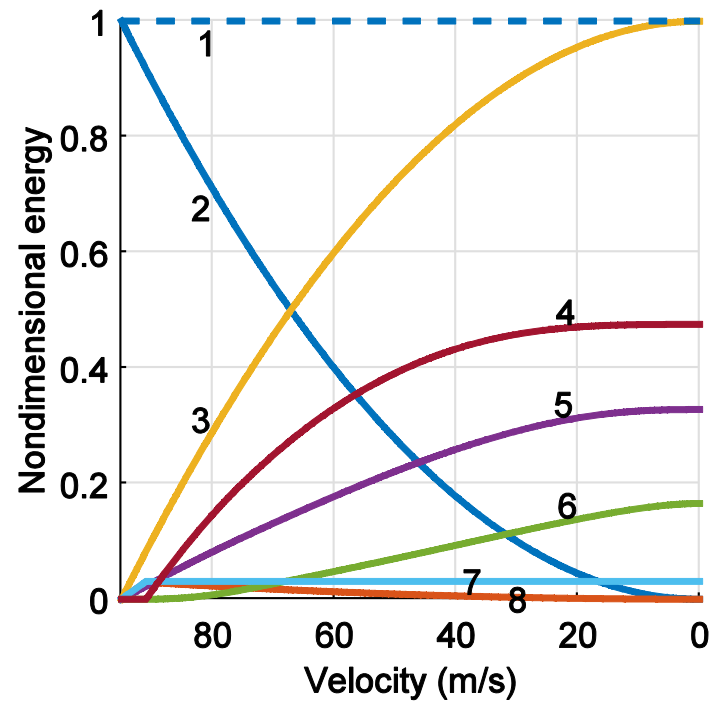

Fig. 10 Nondimensional energy balance at the initial impact velocity of $95 \mathrm{~m} / \mathrm{s}$ as a function of projectile velocity. The individual energies are scaled using the initial kinetic energy of the projectile. Legend numbers 1 initial kinetic energy, 2 projectile kinetic energy, 3 total work done by penetration resistance, 4 energy dissipation due to target material inertia, 5 energy dissipation due to crushing, 6 energy dissipation due to friction, 7 energy transfer to crushed mass moving with the projectile, 8 crushed mass kinetic energy

these tests. The exact values of the utilized parameters are omitted from this work but can found in "Compaction, Shear and Penetration Resistance of LECA" section of Ref. [1]. The parameters related to the frictional resistance were not changed although the results presented in Ref. [1] indicate that also these values differ slightly for the different grain size distributions. The calculated final penetration depths for all of the tests are presented together with the measurements in Fig. 11. Note, that the tests 17-21 were performed using larger projectiles than the tests $1-16$ and 22-26, as mentioned in "Compaction, Shear and Penetration Resistance of LECA" section. The parameters in the calculations were the same for all scales.

The calculated penetration depths correspond reasonably well to the experimental data also for the larger grain size. The calculated penetration depths for the tests with the larger projectiles almost coincide as the projectiles were nearly geometrically similar. The length-to-diameter ratio of the smallest projectile used in the majority of the tests was greater than that of the other two projectiles. The proportionally larger projectile lateral area gives rise to proportionally greater frictional resistance in the model, which in turn decreases the scaled penetration depth.

\section{Sensitivity to Parameter Variation}

The sensitivity of the results is evaluated by independent variations of the input parameters. The parameters 


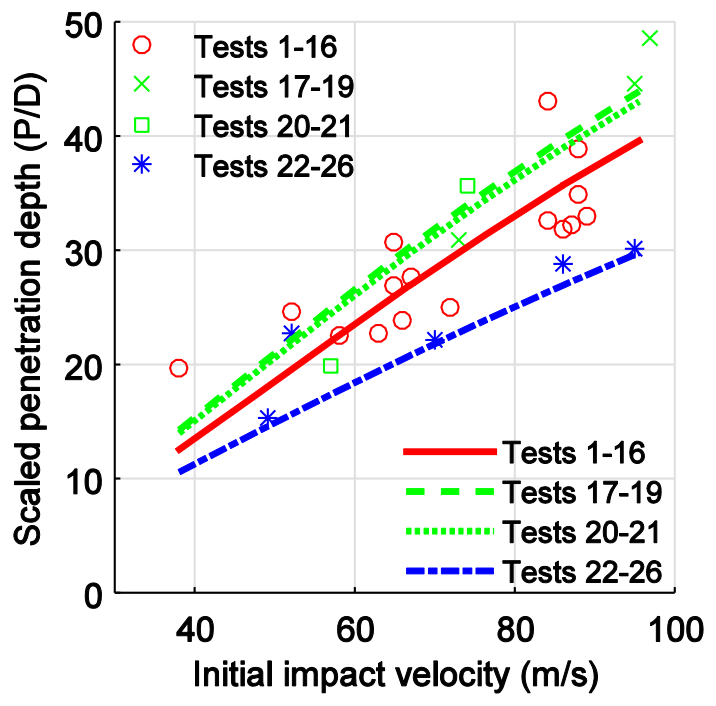

Fig. 11 Nondimensional penetration depths for the full experimental programme (individual markers) and the corresponding results calculated with the developed model (continuous lines)

presented in Table 2 are changed such that each of the resistance component of the model (inertial, crushing and friction) increases or decreases by $20 \%$ with respect to the value presented in the table. In addition, the effect of the amount of the crushed mass sticking to the projectile front is evaluated. The preceding calculations were performed with the assumption that a hemispherical nose is formed in front of the projectile. In the model, the accumulating nose acts by increasing the mass in motion. Therefore, the behaviour of the model is studied for cases where the crushed mass accumulation is five times the base value $(+400 \%)$ and no crushed mass sticks to the projectile front $(-100 \%)$. The effect of parameter variations on the calculated final penetration depth in the 8 sensitivity analyses for the LECA KS04 experiments (tests 22-26 of Fig. 4) are presented in Table 4. The presented values are the minimum, maximum and average differences of calculations covering the initial impact velocity range from 40 to $100 \mathrm{~m} / \mathrm{s}$.

The sensitivities presented in Table 4 indicate expected results. The increase of parameters that increase frictional or compaction resistance decreases the penetration depth. The inertial resistance is governed by the density of the target material and increasing the density decreases the predicted penetration depth. The relative effects of the resistance components are similar over the studied initial impact velocity range but the inertial resistance is the most velocity-dependent. A significant amount of crushed mass accumulation in front of the projectile has a considerable effect on the penetration. This is caused by the mass sticking with the projectile that increases the effective mass in motion of the projectile-crushed mass system and the lesser kinetic energy dissipation yields to greater penetration depths. The kinetic energy of the crushed mass not sticking with the projectile is lost from the primary body in motion.

\section{Discussion and Conclusions}

This paper proposes a penetration model derived from the deformation mechanisms of the studied granular LECA material and the mass and momentum balance considerations of the projectile and penetrated material. The model includes contributions from the inertia, crushing and compaction and frictional resistance of the penetrated material. The model was calibrated and validated using the experimental data from the laboratory scale penetration tests performed using a blunt-nosed steel projectile and crushable granular LECA material. The considerations of typical forms of the Newton's second law based penetration models indicated that the measured penetration response in the studied case cannot be obtained with constant parameter values. In addition, the parameters for this type of models calibrated with the penetration experiments alone are typically not the explicit physical properties of the penetrated material, but dependent also on the projectile and the experimental conditions. In the developed model, most of the parameters relate to the physical properties of the penetrated material and only the parameters related to the interaction between the projectile and penetrated material were calibrated based on the penetration experiments.

Table 4 Sensitivity of the nondimensional penetration depth with respect to the change in the model input parameters

\begin{tabular}{|c|c|c|c|c|c|c|c|c|}
\hline \multirow[b]{2}{*}{ Parameter change } & \multicolumn{2}{|c|}{ Friction $(\%)$} & \multicolumn{2}{|c|}{ Compaction $(\%)$} & \multicolumn{2}{|c|}{ Target density $(\%)$} & \multicolumn{2}{|c|}{ Crushed mass accumulation $(\%)$} \\
\hline & -20 & +20 & -20 & +20 & -20 & +20 & -100 & +400 \\
\hline Min & 5.2 & -6.1 & 4.6 & -6.9 & 4.3 & -7.7 & -3.0 & 8.5 \\
\hline $\operatorname{Max}$ & 7.6 & -4.5 & 5.6 & -6.0 & 9.4 & -3.9 & -2.7 & 11.0 \\
\hline Average & 6.4 & -5.3 & 5.1 & -6.4 & 7.1 & -6.0 & -2.9 & 10.1 \\
\hline
\end{tabular}

The change is given as a signed difference in percentages compared to the line corresponding to calculations of tests $22-26$ of Fig. 11 Positive values indicate greater penetration depths when compared to the reference value and negative values indicate smaller penetration depths 
The model predicted the experimentally measured penetration depth and deceleration with reasonable accuracy for the three cases studied in detail. The model also predicted the decrease of the penetration resistance with decreasing projectile velocity correctly although the calculated initial deceleration was somewhat higher than the measured values. This resulted in a slightly faster decrease of the calculated projectile velocity than the experimental data indicated. The model was also tested by calculating 23 other cases where only the measured penetration depths were available. Without recalibrating the interaction parameters of the model, the predicted penetration depths corresponded to the experimental results fairly well.

The idealisations of the penetrated material behaviour made in the derivation were based on experimental findings reported in literature. Some of these findings were also observed in the current experiments. In the experiments, a compacted portion of the crushed material was found in front of the projectile after the penetration. In the model, this effect was accounted by the increase of the mass in motion and the transfer of both the kinetic energy and momentum from the projectile to the crushed and compacted mass traveling with the projectile. This effect was found to increase the penetration depth as the work done to overcome the inertial resistance was not lost from the system.

One of the primary assumptions in the model was that the penetrated material is crushed and compacted exclusively in front of the projectile. This assumption is based on the reported observations of a compaction front independent of the projectile velocity and a damage front in the nose region of the projectile $[8,15,33]$. This feature was included in the model by assuming a constant initial volume for the compacted region and the volume change was controlled by the amount of the projectile displacement within the time increment. The frictional resistance was modelled with the assumption that the material pushed laterally aside by the projectile comes back into contact with the rear section of the projectile. This assumption was justified by the observations made in experiments of projectile penetration into sand targets [7, 8]. For the developed penetration model, this assumption induced a frictional resistance increasing during the early stages of the penetration and later stabilising to a constant value. At the final stages of the penetration, the frictional resistance was the dominating resistance component.

The author considers that the proposed model includes the most essential mechanisms related to the dynamic penetration resistance of the studied granular LECA material. Several issues remain related to the modelling of each of these phenomena. In front of the projectile, some of the particles crushed to fine sand may form a compacted plug while others are dispersed to the wake as was observed in the present experiments and by other authors as well $[15,33]$. In the calculations, it was assumed that the crushed material forms a hemispherical nose to the projectile and the kinetic energy of transferred to the material in the nose is not lost from the system. In the sensitivity analyses, the size of the formed nose was found to be important for the final penetration depth. The more detailed modelling of the accumulation of the crushed mass requires additional measurements of the size and amount of the compacted plug and crushed particles. In the proposed model, it was assumed that a statically determined confined compaction and frictional behaviour are applicable to the dynamic penetration phenomenon. The findings reported in literature [37-40] indicate that both of these features may be dependent on the loading rate and other loading conditions. Dynamic compaction, shear and skin friction experiments would address this issue. The modelling of the frictional resistance was motivated by the experimental findings of a non-uniform lateral pressure acting on the projectile $[8,15]$, but the assumed simplified form essentially averages the clearly velocity-dependent lateral pressure effect. A considerable further improvement would be the phenomenological modelling of the laterally displaced penetrated material and the resulting lateral pressure on the projectile. The outcome would be a friction model that accounts for the velocity of the projectile such that the frictional resistance may increase as the projectile velocity decreases.

Acknowledgments The preparation of this paper was supported by the VTT Technical Research Centre of Finland. The experimental programme was funded by Posiva Oy. The author wishes to thank the people who contributed to the experimental programme and those who assisted in the preparation of the manuscript as well as the reviewers for their valuable comments.

\section{References}

1. Kuutti J, Hakola I, Fortino S (2012) Analyses of Disposal Canister Falling Accidents. Report ID POSIVA 2012-36. Posiva Oy. ISBN 9789516522176. http://www.posiva.fi/files/3115/ POSIVA_2012-36.pdf

2. Kukkola T (2005) Shafrt shock absorber for a spent fuel canister. In: Proceedings of 18th international conference on structural mechanics in reactor technology (SMiRT 18), pp 4697-4711

3. Liew JYR, Sohel KMA, Koh CG (2009) Impact tests on steelconcrete-steel sandwich beams with lightweight concrete core. Eng Struct 31:2045-2059. doi:10.1016/j.engstruct.2009.03.007

4. Sohel KMA, Liew JYR (2014) Behavior of steel-concrete-steel sandwich slabs subject to impact load. J Constr Steel Res 100:163-175. doi:10.1016/j.jcsr.2014.04.018

5. Sohel KMA, Liew JYR, Koh CG (2015) Numerical modelling of lightweight steel-concrete-steel sandwich composite beams subjected to impact. Thin-Walled Struct 94:135-146. doi:10. 1016/j.tws.2015.04.001

6. Børvik T, Dey S, Olovsson L (2015) Penetration of granular materials by small-arms bullets. Int J Impact Eng 75:123-139. doi:10.1016/j.ijimpeng.2014.07.016 
7. Borg JP, Morrissey MP, Perich CA, Vogler TJ, Chhabildas LC (2013) In situ velocity and stress characterization of a projectile penetrating a sand target: experimental measurements and continuum simulations. Int J Impact Eng 51:23-35. doi:10.1016/j. ijimpeng.2012.07.009

8. Collins AL, Addiss JW, Walley SM, Promratana K, Bobaru F, Proud WG et al (2011) The effect of rod nose shape on the internal flow fields during the ballistic penetration of sand. Int $\mathbf{J}$ Impact Eng 38:951-963. doi:10.1016/j.ijimpeng.2011.08.002

9. Goldman DI, Umbanhowar P (2008) Scaling and dynamics of sphere and disk impact into granular media. Phys Rev E 77:1-14. doi:10.1103/PhysRevE.77.021308

10. de Bruyn JR, Walsh AM (2004) Penetration of spheres into loose granular media. Can J Phys 82:439-446. doi:10.1139/p04-025

11. Ambroso MA, Kamien RD, Durian DJ (2005) Dynamics of shallow impact cratering. Phys Rev E 72:041305. doi:10.1103/ PhysRevE.72.041305

12. Clark AH, Kondic L, Behringer RP (2012) Particle scale dynamics in granular impact. Phys Rev Lett 109:238302. doi:10. 1103/PhysRevLett.109.238302

13. Ciamarra MP, Lara AH, Lee AT, Goldman DI, Vishik I, Swinney HL (2004) Dynamics of drag and force distributions for projectile impact in a granular medium. Phys Rev Lett 92:194301. doi:10. 1103/PhysRevLett.92.194301

14. Riera JD (1968) On the stress analysis of structures subjected to aircraft impact forces. Nucl Eng Des 8:415-426. doi:10.1016/ 0029-5493(68)90039-3

15. Omidvar M, Iskander M, Bless S (2014) Response of granular media to rapid penetration. Int J Impact Eng 66:60-82. doi:10. 1016/j.ijimpeng.2013.12.004

16. Allen WA, Mayfield EB, Morrison HL (1957) Dynamics of a projectile penetrating sand. J Appl Phys 28:370. doi:10.1063/1. 1722750

17. Backman ME, Goldsmith W (1978) The mechanics of penetration of projectiles into targets. Int J Eng Sci 16:1-99. doi:10. 1016/0020-7225(78)90002-2

18. Weber Saint Gobain (2016) Leca ${ }^{\circledR}$ UK Lightweight AggregateWeber 2016. http://www.netweber.co.uk/lecar-uk-lightweightaggregate.html. Accessed 21 Jul 2016

19. CEN (European Commitee for Standardization) (2004) European standard EN 13055-1:2002 "Lightweight aggregates. Part 1: lightweight aggregates for concrete, mortar and grout" and its corrigendum

20. CEN (European Commitee for Standardization) (2004) Technical specification CEN ISO/TS 17892-10:2004 "Geotechnical investigation and testing". Laboratory testing of soil. Part 10: direct shear tests

21. Umbanhowar P, Goldman DI (2010) Granular impact and the critical packing state. Phys Rev E 82(1):010301. doi:10.1103/ PhysRevE.82.010301

22. Clark AH, Behringer RP (2013) Granular impact model as an energy-depth relation. EPL 101:64001. doi:10.1209/0295-5075/ $101 / 64001$

23. Bless S, Peden B, Guzman I, Omidvar M, Iskander M (2014) Dynamic behavior of materials, vol 4. Springer, Cham. doi:10. 1007/978-3-319-00771-7
24. Katsuragi H, Durian DJ (2013) Drag force scaling for penetration into granular media. Phys Rev E 87:052208. doi:10.1103/Phys RevE.87.052208

25. Uehara JS, Ambroso MA, Ojha RP, Durian DJ (2003) Low-speed impact craters in loose granular media. Phys Rev Lett 90:194301. doi:10.1103/PhysRevLett.90.194301

26. Forrestal MJ, Luk VK (1992) Penetration into soil targets. Int J Impact Eng 12:427-444. doi:10.1016/0734-743X(92)90167-R

27. Macek RW, Duffey TA (2000) Finite cavity expansion method for near-surface effects and layering during earth penetration. Int J Impact Eng 24:239-258. doi:10.1016/S0734-743X(99)00156-6

28. Scheffler DR, Zukas JA (2000) Practical aspects of numerical simulation of dynamic events: material interfaces. Int J Impact Eng 24:821-842. doi:10.1016/S0734-743X(00)00003-8

29. Pan J, Selby A (2002) Simulation of dynamic compaction of loose granular soils. Adv Eng Softw 33:631-640. doi:10.1016/ S0965-9978(02)00067-4

30. Borg JP, Vogler TJ (2008) Mesoscale simulations of a dart penetrating sand. Int J Impact Eng 35:1435-1440. doi:10.1016/j. ijimpeng.2008.07.064

31. Oñate E, Rojek J (2004) Combination of discrete element and finite element methods for dynamic analysis of geomechanics problems. Comput Methods Appl Mech Eng 193:3087-3128. doi:10.1016/j.cma.2003.12.056

32. Abbas H, Paul DK, Godbole PN, Nayak GC (1995) Soft missile impact on rigid targets. Int $\mathrm{J}$ Impact Eng 16:727-737. doi:10. 1016/0734-743X(95)00004-T

33. Van Vooren A, Borg J, Sandusky H, Felts J (2013) Sand penetration: a near nose investigation of a sand penetration event. Procedia Eng 58:601-607. doi:10.1016/j.proeng.2013.05.069

34. Potyondy JG (1961) Skin friction between various soils and construction materials. Géotechnique 11:339-353. doi:10.1680/ geot.1961.11.4.339

35. Brumund WF, Leonards GA (1973) Experimental study of static and dynamic friction between sand and typical construction materials. J Test Eval 1:162-165

36. Uesugi M, Kishida $H$ (1986) Frictional resistance at yield between dry sand and mild steel. Soils Found 26:139-149. doi:10. 3208/sandf1972.26.4_139

37. Andrade JE, Chen Q, Le PH, Avila CF, Evans TM (2012) On the rheology of dilative granular media: bridging solid- and fluid-like behavior. J Mech Phys Solids 60:1122-1136. doi:10.1016/j.jmps. 2012.02.011

38. Jackson JG Jr, Ehrgott JQ, Rohani B (1980) Loading rate effects on compressibility of sand. $\mathrm{J}$ Geotech Geoenviron Eng 106:839-852

39. Omidvar M, Iskander M, Bless S (2012) Stress-strain behavior of sand at high strain rates. Int J Impact Eng 49:192-213. doi:10. 1016/j.ijimpeng.2012.03.004

40. Song B, Chen W, Luk V (2009) Impact compressive response of dry sand. Mech Mater 41:777-785. doi:10.1016/j.mechmat.2009. 01.003 